\title{
PAIR-PACKINGS AND PROJECTIVE PLANES
}

\author{
D. R. STINSON \\ (Received 30 November 1982; revised 21 March 1983) \\ Communicated by W. D. Wallis
}

\begin{abstract}
An $\left(n+1, n^{2}+n+1\right)$-packing is a collection of blocks, each of size $n+1$, chosen from a set of size $n^{2}+n+1$, such that no pair of points is contained in more than one block. If any two blocks contain a common point, then the packing can be extended to a projective plane of order $n$, provided the number of blocks is sufficiently large. We study packings which have a pair of disjoint blocks (such a packing clearly cannot be extended to a projective plane of order $n$ ). No such packing can contain more than $n^{2}+n / 2$ blocks. Also, if $n$ is the order of a projective plane, then we can construct such a packing with $n^{2}+1$ blocks.
\end{abstract}

1980 Mathematics subject classification (Amer. Math. Soc.): 05 B 25, 05 B 40.

\section{Introduction}

Let $X$ be a set of $v$ elements called points. A $(k, v)$-packing (based on $X)$ is a collection of $k$-subsets of $X$ (blocks or lines), such that every pair $\left\{x_{1}, x_{2}\right\} \subseteq X$ is contained in at most one block. Equivalently, we require that $\left|B_{1} \cap B_{2}\right| \leqslant 1$ for all distinct blocks $B_{1}$ and $B_{2}$. The packing number $D(k, v)$ is defined to be the largest number of blocks in any $(k, v)$-packing.

We may define projective plane of order $n$ to be any $\left(n+1, n^{2}+n+1\right)$-packing with $n^{2}+n+1$ blocks. It is well known that any two blocks in a projective plane contain a common point, and, dually, every pair of points is contained in a block. Hence $D\left(n+1, n^{2}+n+1\right) \leqslant n^{2}+n+1$, with equality occurring if and only if there exists a projective plane of order $n$.

Suppose $P$ is any $\left(n+1, n^{2}+n+1\right)$-packing. We say that $P$ is extendible if one can construct a projective plane of order $n$ by adding sufficiently many

(C) 1984 Australian Mathematical Society $0263-6115 / 84 \$ A 2.00+0.00$ 
blocks to $P$. When is an $\left(n+1, n^{2}+n+1\right)$-packing extendible? An obvious necessary condition is that any two blocks of $P$ must contain a common point. (We say that such a packing is a partial projective plane of order $n$.) This necessary condition is also sufficient, provided $P$ contains enough blocks. We state this precisely as

TheOREM 1.1 (Dow [2]). A partial projective plane of order $n$ with more than $n^{2}-2 \sqrt{n+3}+6$ blocks is extendible.

On the other hand, suppose an $\left(n+1, n^{2}+n+1\right)$-packing $P$ contains a pair of disjoint blocks. We say that $P$ is a pseudo -partial projective plane (of order $n$ ), which we abbreviate to PPPP. Of course, no PPPP is extendible.

In this paper, we study the function $\operatorname{PPPP}(n)$, which denotes the maximum number of blocks in a pseudo-partial projective plane of order $n$. We show that $\operatorname{PPPP}(n)<n^{2}+n / 2$ for $n>2$. Also, if there exists a projective plane of order $n$, then $\operatorname{PPPP}(n) \geqslant n^{2}+1$. For $2 \leqslant n \leqslant 5, \operatorname{PPPP}(n)=n^{2}+1$. The first unknown value is $\operatorname{PPPP}(6)$. Here we know only that $32 \leqslant \operatorname{PPPP}(6)=D(7,43) \leqslant 38$.

We also investigate the existence of PPPP's in which every point occurs in either $n$ or $n+1$ blocks. We know of only two such packings. Various conditions necessary for existence are obtained, and certain small possibilities are shown not to exist. The smallest unknown case occurs when $n=5$ and would have 17 blocks. This packing is equivalent to a resolvable group-divisible design on 20 points with group-size 2 and block-size 4.

\section{An upper bound for $\operatorname{PPPP}(n)$}

Suppose we have an $\left(n+1, n^{2}+n+1\right)$-packing. A block is said to be spanning if it meets every other block in a point. If every block is spanning, the packing is a partial projective plane of order $n$. If however, there exists a non-spanning block, then the packing is a pseudo-partial projective plane. The number of blocks $b=n^{2}+n+1-\alpha$ for some $\alpha>0$; we will use the notation $(n, \alpha)$-PPPP to describe such a packing.

For a point $x$, the degree of $x$, is the number $r_{x}$ of blocks in which $x$ occurs (note that $r_{x} \leqslant n+1$ for all $x$ ).

LEMMA 2.1. Suppose $B$ is a non-spanning block in an ( $n, \alpha)$-PPPP. Then every point on $B$ has degree at most $n$. Hence $B$ meets at most $n^{2}$ blocks, and $B$ is disjoint from at least $n+1-\alpha$ blocks. 
Proof. Suppose $x \in B$ has $r_{x}=n+1$. Let $B_{1}$ be a block disjoint from $B$. The point $x$ occurs on a block with every other point so there are $n+1$ lines joining $x$ to points on $B_{1}$. But $x$ also occurs on $B$, so $r_{x}>n+1$, an impossibility.

COROLlary 2.2. In an (n, $\alpha)$-PPPP, there are at least $\frac{1}{2}(n+2-\alpha)(n+1-\alpha)$ unordered pairs of disjoint blocks.

We now obtain an upper bound for the number of pairs of disjoint blocks.

LEMMA 2.3. The number of pairs of disjoint lines in an $(n, \alpha)-\operatorname{PPPP}$ is $\left(\begin{array}{c}b \\ 2\end{array}\right)-\Sigma_{x}\left(\begin{array}{c}r_{x} \\ 2\end{array}\right)$.

PROOF. The number of pairs of lines meeting in a given point is $\left(\begin{array}{c}r_{x} \\ 2\end{array}\right)$. Summing over $x$, we obtain the number of pairs of non-disjoint lines. This quantity is subtracted from the total number of pairs of lines $\left(\begin{array}{c}b \\ 2\end{array}\right)$, to obtain the desired result.

LEMMA 2.4. In an $(n, \alpha)$-PPPP, $\Sigma_{x}\left(\begin{array}{c}r_{x} \\ 2\end{array}\right) \geqslant\left(\begin{array}{c}n+1 \\ 2\end{array}\right)\left(n^{2}+n+1-2 \alpha\right)$, with equality occurring if and only if every point has degree $n$ or $n+1$.

Proof. We have $\Sigma_{x} 1=n^{2}+n+1$ and $\Sigma_{x} r_{x}=(n+1) b$. We write $(n+1) b$ $=\left(n^{2}+n+1\right) n+n^{2}+n+1-\alpha(n+1)$. By the convexity of binomial coefficients, $\Sigma\left({ }_{2}^{r_{x}}\right)$ is minimized when $n^{2}+n+1-\alpha(n+1)$ points have degree $n+1$ and the remainder have degree $n$. The result follows.

From Lemmata 2.3 and 2.4 we calculate

COROLlaRY 2.5. In an (n, $\alpha)$-PPPP, the number of pairs of disjoint lines is at most $\left(\begin{array}{l}\alpha \\ 2\end{array}\right)$, with equality occurring if and only if all points have degree $n$ or $n+1$.

THEOREM 2.6. In an $(n, \alpha)$-PPPP, $\alpha \geqslant(n+2) / 2$.

Proof. From Corollaries 2.2 and $2.5,\left({ }^{n+2-\alpha}\right) \leqslant\left(\frac{\alpha}{2}\right)$, which simplifies to the desired inequality.

COROLLARY 2.7. If there is no projective plane of order $n$, then $D(n+1$, $\left.n^{2}+n+1\right) \leqslant n^{2}+n / 2$.

Proof. Theorems 1.1 and 2.6.

We now examine the case $\alpha=(n+2) / 2$ for PPPPs. All points must have degree $n$ or $n+1$; there are $\left(\begin{array}{c}n+2 \\ 2\end{array}\right)$ points of degree $n$ and $\left(\begin{array}{c}n \\ 2\end{array}\right)$ points of degree 
$n+1$, from the proof of Lemma 2.4. We have $(n+1) / 2$ non-spanning lines, which are mutually disjoint. Consider the dual incidence structure: it has $n^{2}+n / 2$ points, and $n^{2}+n+1$ blocks (of lengths $n$ and $n+1$ ). All pairs occur in a unique block, except for $(n+2) / 2$ "independent" points. If we adjoin a block consisting of these $(n+2) / 2$ points, then we have a pairwise balance design (PBD) with $n^{2}+n / 2$ points and $n^{2}+n+2$ blocks. For $n \geqslant 3$, such a PBD does not exist, by [5]. For $n=2$ one can construct a $(2,2)$-PPPP. The blocks are: 123 , 456, 147, 257, 267. This discussion implies

ThEOREM 2.8. For $n \geqslant 3$, there is no $(n,(n+2) / 2)$-PPPP. Also, there exists (2, 2)-PPPP.

COROLlary 2.9. If there does not exist a projective plane of order $n$, then $D\left(n+1, n^{2}+n+1\right)<n^{2}+n / 2$.

Proof. In view of Theorem 2.8 and Corollary 2.7, it suffices to note that there is a projective plane of order 2 .

\section{Some values of $\operatorname{PPPP}(n)$}

Recall that $\operatorname{PPPP}(n)$ denotes the largest number of blocks in a pseudo-partial projective plane of order $n$. From the previous section, we have

LemMA 3.1. $\operatorname{PPPP}(2)=5$, and for all $n \geqslant 3, \operatorname{PPPP}(n)<n^{2}+n / 2$.

We provide a lower bound for values of $n$ where a projective plane of order $n$ exists.

LEMMA 3.2. If there is a projective plane of order $n$, then $\operatorname{PPPP}(n) \geqslant n^{2}+1$.

ProOF. Let $\mathscr{Q}$ be an affine plane of order $n$, with parallel classes $\mathscr{P}_{1}, \ldots, \mathscr{P}_{n+1}$, and let $\left\{\infty_{1}, \ldots, \infty_{n+1}\right\}$ be a set of $n+1$ new points. For $1 \leqslant i \leqslant n$ adjoin the point $\infty_{i}$ to each line of the class $\mathscr{P}_{i}$. Now replace the point $\infty_{1}$ by $\infty_{n+1}$ in some block of $\mathscr{P}_{1}$, delete all lines in $\mathscr{P}_{n+1}$, and add a new block $\left\{\infty_{1}, \infty_{2}, \ldots, \infty_{n+1}\right\}$.

EXAMPLE 3.3. A $(3,3)$-PPPP.

$$
\begin{array}{lll}
\infty_{1} 123 & \infty_{2} 159 & \infty_{3} 147 \\
\infty_{1} 456 & \infty_{2} 267 & \infty_{3} 258 \\
\infty_{4} 789 & \infty_{2} 348 & \infty_{3} 369 \\
\infty_{1} \infty_{2} \infty_{3} \infty_{4} & &
\end{array}
$$


Corollary 3.4. $\operatorname{PPPP}(3)=10$ and $\operatorname{PPPP}(4)=17$.

Proof. Lemmata 3.1 and 3.2.

For $n=5$, we have $26 \leqslant \operatorname{PPPP}(5) \leqslant 27$ by Lemmata 3.1 and 3.2. We will show that there is no $(5,4)$-PPPP; hence $\operatorname{PPPP}(5)=26$.

Suppose we have a $(5,4)$-PPPP. Let $X$ denote the set of points and $B$ the set of blocks. A spanning block in $\mathscr{B}$ must contain at least two points of degree six. Consider the incidence structure formed by the set of points $Y$ of degree six. The blocks are $\mathcal{C}=\{Y \cap B: B \in \mathscr{B}$ and $B$ is spanning $\}$. Every pair of points in $Y$ occurs in a unique block of $\mathcal{C}$, and every point in $Y$ occurs in six blocks in $C$. Also, each block in $\mathcal{C}$ has size at least two. Let $z \in X \backslash Y$. Then $\mathfrak{P}_{z}=\{B \cap Y$ : $z \in B \in \mathscr{B}\}$ is a set of blocks in $\mathcal{C}$ which forms a partition of $Y$.

Let $p=|Y|, q=|\mathcal{E}|$. For $\mathscr{B}$ to contain a pair of (non-spanning) disjoint blocks, $q \leqslant 25$ and $p \leqslant 19$.

LEMMA 3.5. C contains only blocks of size $2,3,4$.

Proof. Suppose $C \in \mathcal{C}$ has size at least 5. Then $C=B \cap Y$ where $B \in \mathscr{B}$. The number of blocks spanned by $B$ is $27=1+\sum_{x \in B}\left(r_{x}-1\right)$, from which it follows that $B$ contains five points of degree 6 and one point $z$ of degree 2 . Thus $|C|=5$. Now $\mathscr{P}_{z}=\{C, D\}$, say, where $C \cup D=Y$. Thus $\mathcal{C}$ has blocks $C, D$ and $|C| \cdot|D|$ further blocks, each containing one point from $C$ and one from $D$. Since each point of $Y$ has degree six, we must have $|d|=5$, whence $q=27>25$, a contradiction.

Let $b_{i}$, for $i=2,3,4$, denote the number of blocks of size $i$ in $\mathcal{C}$. Elementary counting yields

$$
\left(\begin{array}{lll}
1 & 1 & 1 \\
1 & 3 & 6 \\
2 & 3 & 4
\end{array}\right)\left(\begin{array}{l}
b_{2} \\
b_{3} \\
b_{4}
\end{array}\right)=\left(\begin{array}{c}
q \\
\frac{p(p-1)}{2} \\
6 p
\end{array}\right) .
$$

The coefficient matrix is non-singular, so we may solve for $b_{2}, b_{3}$, and $b_{4}$ obtaining

LEMMA 3.6.

$$
\left(\begin{array}{l}
b_{2} \\
b_{3} \\
b_{4}
\end{array}\right)=\left(\begin{array}{ccc}
6 & 1 & -3 \\
-8 & -2 & 5 \\
3 & 1 & -2
\end{array}\right)\left(\begin{array}{c}
q \\
\frac{p(p-1)}{2} \\
6 p
\end{array}\right) .
$$


LEMMA 3.7. $7 \leqslant p \leqslant 10$.

Proof. First, note that $p \geqslant 7$, since any point in $Y$ occurs with at least one other point in $Y$ in each block containing it. Also, we have noted that $p \leqslant 19$.

The condition $b_{2} \geqslant 0$ gives $0 \leqslant 6 q+p(p-37) / 2$ (from Lemma 3.6). Since $q \leqslant 25$, we have $p(p-37) \leqslant 30$. Thus $p \leqslant 12$ or $p \geqslant 25$. Similarly $b_{4} \geqslant 0$ yields $p \leqslant 10$ or $p \geqslant 15$.

Combining all those inequalities, the desired result is obtained.

LEMMa 3.8. $p \neq 7$ or 8 .

Proof. If $p=7$ then all blocks are of size 2. But then there is no way to find a set of blocks of $C$ that forms a partition of $Y$.

If $p=8$, then every point of $Y$ occurs (in $C$ ) in one block of size 3 and five blocks of size 2 . Then $b_{3}=8 / 3$, which is absurd.

LEMMA 3.9. $p \neq 9$.

Proof. If $p=0$, we would have $b_{2}+3 b_{3}+6 b_{4}=36$ and $2 b_{3}+3 b_{3}+4 b_{4}=$ 54. This implies $3 b_{3}+8 b_{4}=18$. Since $b_{3}$ and $b_{4}$ are non-negative integers, $b_{3}=6$ and $b_{4}=0$; whence $b_{2}=18$.

Let $z \in X \backslash Y$. Then $\mathscr{P}_{z}$ must contain at least one block of size 3 . The six blocks in $\mathcal{C}$ of size 3 contain at most eighteen points of $X \backslash Y$. But $|X \backslash Y|=31-p=22$ $>18$. This is a contradiction.

LEMMA 3.10. $p \neq 10$.

Proof. We have $b_{2}+3 b_{3}+6 b_{4}=45$ and $2 b_{2}+3 b_{3}+4 b_{4}=60$; hence $3 b_{3}+$ $8 b_{4}=30$. Thus $\left(b_{3}, b_{4}\right)=(2,3)$ or $(10,0)$.

In the first case, $b_{2}=21$ and $q=26$, an impossibility.

In the second case $b_{2}=15$ and $q=25$. Now $X$ must contain a point $z$ of degree at most 4 , for $(27 \cdot 6-10 \cdot 6) / 21<5$. Let $C \subseteq B \in 9 B$. $B$ is spanning, so $\Sigma_{x \in B} r_{x}=32$. Suppose $B$ contains two points of degree 6 , so the remaining four points in $B$ have degree 5. It follows that $z \notin B$. Thus $\mathscr{P}_{z}$ contains no blocks of size 2. But this is impossible, since 3 does not divide 10 .

As a result of Lemmata $3.7-3.10$, we have

LEMma 3.11. There is no (5,4)-PPPP. Hence PPPP $(5)=26$. 
For $n=6$, we know much less. In [4], it is shown that the largest partial projective plane of order 6 has at most 27 blocks. We can construct a $(6,11)$ PPPP. This implies $32 \leqslant \operatorname{PPPP}(6)=D(7,43)$.

LEMMa 3.12. There exists a $(6,11)$-PPPP.

Proof. In [1], Baker shows that $D(7,45) \geqslant 45$. Every point in this packing occurs in seven blocks. Let $B$ be a block, and let $x, y$ be points in $B$. Then the 32 blocks which contain neither $x$ nor $y$ form a $(7,43)$-packing.

It can easily be seen that this new packing has many pairs of disjoint lines, so we have a $(6,11)$-PPPP.

From Lemma 3.1, PPPP $(7) \leqslant 38$. Thus we have

LEMMA 3.13. $32 \leqslant D(7,43)=\operatorname{PPPP}(7) \leqslant 38$

\section{Quasi-regular PPPPs}

Suppose we have a pseudo-partial projective plane in which every point has degree $n$ or $n+1$. We call such a packing a quasi-regular PPPP, which we abbreviate to QRPPPP. We can determine several necessary conditions for the existence of QRPPPP. Indeed, at the time of this writing, only a few examples are known. From a previous section, we have

LEMMA 4.1. If an $(n, \alpha)$-QRPPPP exists, then $\alpha \geqslant(n+2) / 2$. Further, $\alpha=$ $(n+2) / 2$ can hold only for $n=2$.

The following upper bound is easily proved.

LEMMA 4.2. If an ( $n, \alpha)$-QRPPPP exists, then $\alpha \leqslant n$.

Proof. Suppose $\alpha \geqslant n+1$. Any block $B$ meets at least $n^{2}$ blocks. But the number of blocks $b \leqslant n^{2}$, so $B$ is spanning. Since $B$ is an arbitrary block, we have a partial projective plane, a contradiction.

We now derive several results concerning QRPPPP. All these follow from simple counting arguments. 
LEMMA 4.3. In an ( $n, \alpha)$-QRPPPP, a non-spanning block contains only points of degree $n$, whereas a spanning block contains $n+1-\alpha$ points of degree $n+1$ and $\alpha$ points of degree $n$.

LEMMA 4.4. In an $(n, \alpha)$-QRPPPP, a non-spanning block is disjoint from precisely $n+1-\alpha$ blocks.

LEMMA 4.5. In an ( $n, \alpha)$-QRPPPP, there are $\alpha(n+1)$ points of degree $n$ and $n^{2}+n+1-\alpha(n+1)$ points of degree $n+1$.

Proof. Let there be $x$ points of degree $n+1$. Counting incident point-block pairs, we obtain $\left(n^{2}+n+1-\alpha\right)(n+1)=n\left(n^{2}+n+1\right)+x$.

LEMMA 4.6. In an $(n, \alpha)$-QRPPPP, there are $\left(\alpha^{2}-\alpha\right) /(n+1-\alpha)$ non-spanning blocks and $(n+1)\left(n^{2}+n+1-\alpha(n+1)\right) /(n+1-\alpha)$ spanning blocks.

Let the number of non-spanning blocks be denoted by $x$. Count pairs of disjoint blocks:

$$
\frac{x(n+1-\alpha)}{2}=\left(\begin{array}{l}
b \\
2
\end{array}\right)-\Sigma\left(\begin{array}{c}
r_{x} \\
2
\end{array}\right)=\frac{\alpha^{2}-\alpha}{2}
$$

LEMMA 4.7. In an ( $n, \alpha)$-QRPPPP, a point of degree $n+1$ occurs only in spanning blocks, whereas a point of degree $n$ occurs in $(\alpha-1) /(n+1-\alpha)$ non-spanning blocks and $n-(\alpha-1) /(n+1-\alpha)$ spanning blocks.

Proof. A non-spanning block contains only points of degree $n$, so no point of degree $n+1$ occurs on a non-spanning block.

Now, let $x$ denote the number of spanning lines on which a point $y$ of degree $n$ occurs. Every point of degree $n+1$ occurs on a block containing $y$. Thus we have $x(n+1-\alpha)=n^{2}+n+1-\alpha(n+1)$, which simplifies to the desired expression.

COROLLARY 4.8. If an (n, $\alpha)$-QRPPPP exists, then $\alpha=(t /(t+1)) n+1$ for some positive integer $t$.

Proof. From Lemma 4.7, $t=(\alpha-1) /(n+1-\alpha)$ is an integer. Solve for $\alpha$.

COROLlaRY 4.9. If an $(n, \alpha)-\mathrm{QRPPPP}$ exists for $n$ prime, then $n=\alpha$. 
Proof. From Corollary 4.8, $\alpha=(t /(t+1)) n+1$ for an integer $t$. Since $n$ is prime, $t=0$ or $t=n-1$. If $t=0$, then $\alpha=1$ which contradicts Lemma 4.1. Thus $t=n-1$ and $\alpha=n$.

The case $n=\alpha$ is of particular interest. We can characterize $(n, n)$-QRPPPPs in terms of certain group-divisible designs.

THEOREM 4.10. There exists an $(n, n)-\mathrm{QRPPPP}$ if and only if there exists a resolvable GDD having $n^{2}-n$ points, all groups of size 2 , and all blocks of size $n-1$.

Proof. From the preceding lemmata, we obtain the following facts concerning an $(n, n)$-QRPPPP. There are $n+1$ spanning lines, and $n^{2}-n$ non-spanning lines, which occur in disjoint pairs. There is one point of degree $n+1$ and $n^{2}+n$ points of degree $n$. Dualize, obtaining an incidence structure with $n^{2}+1$ points and $n^{2}+n+1$ blocks. There is one block of length $n+1$, which meets all other blocks, and the remaining blocks have length $n$.

The $n^{2}-n$ points not on the block of length $n+1$ can be partitioned into pairs. These pairs are the only ones which do not occur in some block. If we call these pairs groups, and delete the points on the block of length $n+1$, we obtain a group-divisible design, with the desired parameters. Each point of the block of length $n+1$ induces a parallel class of blocks, so the resultant GDD is resolv able.

This entire process can be reversed, so one can obtain an $(n, n)$-QRPPPP from such a resolvable GDD.

COROLlaRY 4.11. There is $a(3,3)-\mathrm{QRPPPP}$, whereas there is no $(4,4)-\mathrm{QRPPPP}$.

Proof. First, consider the case $n=3$. A 1-factorization of the complete graph $K_{6}$ certainly exists. Call one of the 1-factors groups. The resulting GDD is resolvable, with $6=n^{2}-n$ points, $12=n^{2}+n$ blocks, and blocks of size $2=$ $n+1$. By Theorem 4.10, there exists a $(3,3)$-QRPPPP.

Next, let $n=4$. The resolvable GDD here would have 12 points, and 20 blocks, each of size 3. This GDD would be a near-Kirkman triple system NKTS (12), which is known not to exist [3]. Thus no $(4,4)$-QRPPPP exists.

As far as the author knows, the existence of no other GDD in this class has been determined. 
For $n=6$, we have the additional possibility $=5$. Suppose there is a $(6,5)$ QRPPPP. From the previous counting lemmata we have

LEMMA 4.12. In a (6,5)-QRPPPP there are 28 spanning blocks, each of which contains two points of degree seven. Also, there are 35 points of degree six, each of which lies on four spanning blocks.

Let the eight points of degree seven be denoted $1, \ldots, 8$, and let the remaining points be called $a, b, \ldots$ Each of the $28=\left(\begin{array}{l}8 \\ 2\end{array}\right)$ pairs of points of degree seven is contained in one of the spanning blocks. Any point $x$ of degree six induces a one-factor $F_{x}$ (perfect matching) of the graph $K_{8}$ (on vertex set $1, \ldots, 8$ ).

The 35 one-factors thus produced satisfy the properties:

(1) for $x \neq y,\left|F_{x} \cap F_{y}\right| \leqslant 1$,

(2) if $x$ and $y$ are distinct points which occur in a non-spanning block $B$, then $F_{x} \cap F_{y}=\varnothing$.

(3) every pair $\{i, j\}$ is in five of these $F_{x}$ 's.

Now let $B_{1}$ and $B_{2}$ be disjoint non-spanning blocks. The points of $B_{1}$ (resp. $B_{2}$ ) induce a one-factorization of $K_{8}$, by property (2). These two 1 -factorizations are orthogonal (property (1) above). Thus we have Room square $R\left(B_{1}, B_{2}\right)$.

Pick any edge of $K_{8}$, say $\{i, j\}$. Of the five $F_{x}$ containing $\{i, j\}$, two are determined by the Room square. Then the remaining three are (uniquely) determined by the fact that no edge other than $\{i, j\}$ can be repeated in these five one-factors.

This process can be carried out for any edge of $K_{8}$. If we obtain two one-factors which contain precisely two common edges, we have a contradiction.

There are precisely six inequivalent Room squares of side 7 [6]. We begin with each one, in turn, and obtain a contradiction, as described above. This establishes the non-existence of a $(5,5)-Q R P P P P$.

Figure 1. A Room square of side 7

\begin{tabular}{|l|l|l|l|l|l|l|}
\hline 01 & & 45 & 67 & & & 23 \\
\hline 57 & 02 & & & & 13 & 46 \\
\hline & 56 & 03 & 12 & & 47 & \\
\hline & 37 & & 04 & 26 & & 15 \\
\hline 36 & 14 & 27 & & 05 & & \\
\hline 24 & & & 35 & 17 & 06 & \\
\hline & & 16 & & 34 & 25 & 07 \\
\hline
\end{tabular}


Suppose we start with the Room square $R_{11}$ of Figure 1. From the pair 12, we get two one-factors 12034756 and 12043567 . This forces 12053746,120634 57, and 12073645 . Starting with 06, we have 06132547 and 06172435 , which forces 06123745,06142357 , and 06152734 . The one-factors 12063457 and 06123745 contain precisely two common edges, a contradiction.

For each of the remaining five Room squares, a contradiction is obtained in a similar fashion. This discussion implies

LEMMA 4.13. There does not exist a $(6,5)-\mathrm{QRPPPP}$.

For $n \leqslant 6$, we list all positive integers of the form $\alpha=(t /(t+1)) n+1$ and summarize known information concerning QRPPPP's in Table 1 below.

TABLE 1

Existence of QRPPPPs

\begin{tabular}{cccl}
$n$ & $\alpha$ & Existence & Authority \\
\hline 2 & 2 & yes & Lemma 4.1 \\
3 & 3 & yes & Corollary 4.11 \\
4 & 3 & no & Lemma 4.1 \\
4 & 4 & no & Corollary 4.11 \\
5 & 5 & $?$ & \\
6 & 4 & no & Lemma 4.1 \\
6 & 5 & no & Lemma 4.13 \\
6 & 6 & $?$ &
\end{tabular}

\section{Remarks}

We mention several open problems.

(1) Find lower bounds for $\operatorname{PPPP}(n)$ (and $D\left(n+1, n^{2}+n+1\right)$ in the case where $n$ is not the order of a projective plane).

(2) Determine the existence of non-existence of the GDDs of Theorem 4.10. What if the resolvability condition is dropped?

(3) Find $D(7,43)$.

(4) Define an $\left(n+1, n^{2}+n+1\right)$-packing to be maximal if it is not possible to form a larger packing by adding one more block (that is, any $(n+1)$-subset of points meets some block in more than one point). How many blocks can there be in a maximal packing? ( $R$. Mullin has conjectured that a maximal packing has at least $2 n+1$ blocks.) Also, one can construct a $\operatorname{PPPP}$ with $\operatorname{PPPP}(n)$ blocks that is not maximal? 


\section{References}

[1] R. D. Baker, 'An elliptic semiplane,' J. Combinatorial Theory Ser. A 25 (1978), 193-195.

[2] S. Dow, 'An improved bound for extending partial projective planes,' Discrete Math., to appear.

[3] A. Rosa and A. Kotzig, 'Nearly Kirkman systems,' Proc. Fifth Southeastern Conf. on Combinatorics, Graph Theory and Computing, Boca Raton, La., 1974, pp. 607-614.

[4] P. J. Schellenberg, 'Further results on $V_{p}(7,1)$,' Proc. Eighth Southeastern Conference on Combinatorics, Graph Theory, and Computing, Baton Rouge, La., 1977, pp. 591-604.

[5] D. R. Stinson, 'The non-existence of certain finite linear spaces,' Geom. Dedicata, to appear.

[6] W. D. Wallis, A. P. Street and J. S. Wallis, Combinatorics: Room squares, sum-free sets, Hadamard metrics, (Lecture Notes in Math., vol. 292, Springer-Verlag, Berlin, 1972).

\section{Department of Computer Science \\ University of Manitoba \\ Winnipeg, Manitoba R3T 2N2 \\ Canada}

